\title{
Evaluation du stock de carbone aérien de Piliostigma reticulatum en zone soudanienne du Burkina Faso
}

1TYANO Abdoulaye*, 2YELEMOU Barthélemy, ${ }^{1}$ HIEN Mipro, 2 KOALA Jonas

Université Nazi Boni, Institut du Développement Rural (IDR), Laboratoire des systèmes Naturelles, des Agrosystèmes et de l'Ingénierie de l'Environnement (Sy.N.A.I.E), 01 BP 1091 Bobo-Dioulasso 01,

2Institut de l'Environnement et de Recherches Agricoles (INERA)/Centre National de la Recherche Scientifique et Technologique (CNRST), BP 10 Koudougou, Burkina Faso

${ }^{*}$ Correspondant auteur email : tabdoulayemagloire@gmail.com, miphien@gmail.com, koalajonas@gmail.com, yelbart@hotmail.com

Original submitted on 19th September 2019. Published online at www.m.elewa.org/journals/ on 31st December 2019 https://doi.org/10.35759/JABs.v144.7

\section{RESUME}

Objectif: Depuis 2005, le protocole de Kyoto est entré en vigueur et les projets de boisement et de reboisement dans le cadre du Mécanisme de Développement Propre (MDP) sont devenus éligibles. II est donc indispensable d'établir des équations allométriques pour estimer les biomasses des différentes espèces.

Méthodologie et résultats: L'étude a concerné des plantations de Piliostigma reticulatum (Piliostigma, bauhinia, semellier ; pied de chameau) de divers âges implantés sur un sol ferrugineux en zone nord soudanienne du Burkina Faso. La détermination de la biomasse aérienne a été réalisée par la méthode directe. Les biomasses obtenues par mesure directe et celles issues des modèles ont été soumises à ANOVA. Au bout de 5 ans (respectivement 15 ans), $P$. reticulatum stocke une biomasse aérienne de 3,65 \pm

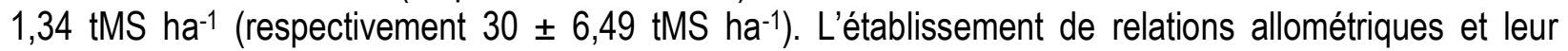
évaluation permet de retenir celles-ci : $Y=10,95 e^{0,062 S H}, Y=-0,514 \mathrm{SH}^{2}+3,724 \mathrm{SH}-0,2313$ et $Y=$ 0,004C+0,002 (respectivement pour les sujets de 15 ans, 05 ans et 03 ans)

Conclusion et application: II existe une forte corrélation entre la quantité de biomasse sèche de $P$. reticulatum et ces mesures dendrométriques. L'espèce possède un fort potentiel de séquestration de carbone. Ces résultats contribuent à la compréhension de l'impact de la plantation d'arbustes et/ ou leur conservation sur le changement climatique.

Mots clés : Carbone, Changements climatiques, Piliostigma reticulatum, relations allométriques, arbustes, Burkina Faso

Assessment of the aerial carbon stock of Piliostigma reticulatum in the Sudanese zone of Burkina Faso 


\begin{abstract}
Objective: Since 2005, the Kyoto Protocol has been in force and afforestation and reforestation projects under the Clean Development Mechanism (CDM) have become eligible. It is therefore essential to establish allometric equations to estimate the biomass of the different species.

Methodology and results: The study concerned $P$. reticulatum plantations of various ages planted on ferruginous soil in the northern Sudanian zone of Burkina Faso. The determination of the above-ground biomass was carried out by the direct method. The biomasses obtained by direct measurement and those from the models were submitted to ANOVA. After 5 years (respectively 15 years), $P$. reticulatum stores an above-ground biomass of $3.65 \pm 1.34$ tMS ha-1 (respectively $30 \pm 6.49$ tMS ha-1). The establishment of allometric relationships and their evaluation makes it possible to retain them: $Y=10.95 \mathrm{e} 0.062 \mathrm{SH}, \mathrm{Y}=$ $0.514 \mathrm{SH} 2+3.724 \mathrm{SH}-0.2313$ and $Y=0.004 \mathrm{C}+0.002$ (respectively for subjects 15,05 and 03 years of age.

Conclusion and application of results: There is a strong correlation between the amount of dry biomass of $P$. reticulatum and these dendrometric measurements. The species has a high potential for carbon sequestration. These results contribute to the understanding of the impact of shrub planting and/or conservation on climate change.
\end{abstract}

Keywords: Carbon, Climate change, Piliostigma reticulatum, allometric relationships, shrubs, Burkina Faso

\section{INTRODUCTION}

Depuis près de deux décennies, le phénomène du réchauffement climatique a pris une ampleur médiatique. Ce réchauffement est dû en grande partie à l'accroissement des concentrations atmosphériques de gaz à effet de serre, essentiellement lié aux activités anthropiques (IPCC (Intergovernmental Panel on Climate Change), 2007). La production de gaz à effet de serre (GES) par l'homme fait planer des menaces sur les écosystèmes terrestres et marins (IPCC, 2007). II est devenu évident que l'augmentation des gaz à effet de serre (GES) dans l'atmosphère et le changement climatique qui en résulte auront des effets majeurs au 21ème siècle (IPCC, 2007). Plusieurs gaz à effet de serre voient leur concentration augmenter. Entre autres on peut citer la vapeur d'eau, le méthane, le soufre, les oxydes d'azote, le monoxyde de carbone (CO), le dioxyde de carbone $\left(\mathrm{CO}_{2}\right)$, etc. Cependant, le dioxyde de carbone $\left(\mathrm{CO}_{2}\right)$ est de loin le plus important gaz à effet de serre anthropique (Hansein, 2004). La concentration dans l'air du $\mathrm{CO}_{2}$ n'a pas été constante au fil des âges (Boulman et al., 2010). En effet, au cours des 400000 dernières années, le taux de $\mathrm{CO}_{2}$ atmosphérique a varié d'environ 180 ppm lors des périodes glacières à $280 \mathrm{ppm}$ pendant les périodes interglaciaires (IPCC, 2007). Par contre, cette concentration s'est mise à augmenter considérablement depuis le début de l'ère industrielle jusqu'à près de 379 ppm en 2005 en raison de l'utilisation de combustibles fossiles et du changement d'utilisation des terres qui ont entrainé une émission considérable de dioxyde de carbone (IPCC, 2007). En effet, les végétaux autotrophes utilisent la photosynthèse pour absorber le $\mathrm{CO}_{2}$ et le transformer en différents composés organiques qui constituent le matériel végétal comme le bois, l'écorce ou les feuilles. La reforestation et la lutte contre la déforestation contribuent donc à la diminution du $\mathrm{CO}_{2}$ dans l'atmosphère (Hansein, 2004).En zone nord soudanienne du Burkina Faso, la végétation ligneuse est principalement composée de Parkia biglobosa (Jacq.) R.Br. ex G. Don, Vitellaria paradoxa Gaertn. f., Faidherbia albida, Lannea microcarpa Engl. et K. Krause, Tamarindus indica L, Khaya senegalensis (Desr.) A. Juss. La strate arbustive est dominée par des fourrés clairsemés de Guiera senegalensis J. F. Gmel., Combretum nigricans Lepr. ex-Guill. et Perr., Piliostigma reticulatum (DC.) Hochst (Yélemou et al., 2007). Cependant au cours des dernières années, dans les parcs agroforestiers de cette zone, les espèces ligneuses arborées telles que Parkia biglobosa, Vitellaria paradoxa, Faidherbia albida, Lannea microcarpa, etc. 
connaissent une régression. Cette régression des espèces ligneuses du milieu se caractérise par leur faible régénération, leur faible densité, ou leur diminution de taille et de plus en plus par un intérêt croissant accordé aux arbustes (Yélemou et al., 2012). C'est ainsi que Piliostigma reticulatum a été adopté par défaut comme espèce agroforestière par les populations du plateau central (Yélemou et al., 2007). Une meilleure investigation sur ces nouveaux choix d'adaptation des populations pourrait garantir leur durabilité. Pour Piliostigma reticulatum, les recherches ont porté entre autres sur: ses potentialités fourragères (Sanou, 2005 ; Ouédraogo, 2006), ses potentialités médicinales (Yélemou et al., 2007), la dynamique de sa

\section{METHODES}

Milieu d'étude : La Station de recherche de l'Institut de I'Environnement et de recherches Agricoles (INERA) à Saria a servi de cadre pour cette étude. Elle est située à $23 \mathrm{~km}$ à l'Est de la ville de Koudougou et à $80 \mathrm{~km}$ au Sud-ouest de Ouagadougou, la capitale du Burkina Faso (Figure 1). Le climat est de type nord soudanien. A Saria, les hauteurs de pluies tombées d'une année à l'autre présentent une grande variabilité. En effet, régénération sexuée (Yélemou et al., 2012), son aptitude à améliorer les propriétés physicochimiques et biologiques du sol (Yélemou et al., 2012 ; Yélemou et al., 2015 ; Tyano et al., 2016), son impact sur la régénération de la végétation ligneuse et herbacée (Tyano, 2016; Yélemou et al., 2017). Cependant, $P$. reticulatum demeure peu connu pour l'élaboration d'une stratégie efficace de sa conservation. En particulier, il existe peu de données scientifiques sur son potentiel de séquestration de carbone en zone soudanienne du Burkina Faso. II en est de même des relations allométriques spécifiques à $P$. reticulatum. C'est pour pallier cette insuffisance, que la présente étude a été entreprise.

celles-ci sont comprises entre 600 et $900 \mathrm{~mm}$ avec une moyenne de 868,67 $\pm 109,41 \mathrm{~mm}$ pour les dix dernières années. Le terroir de Saria, à l'image du Plateau Central est une zone très peuplée. La province du Bulkièmdé, à laquelle appartient Saria, a une densité de $152 \mathrm{hab} . / \mathrm{km}^{2}$ alors que celle de la région du centreOuest est de 54,5 hab. $/ \mathrm{km}^{2}$ (MEF, 2009) et la moyenne nationale est de $37 \mathrm{hab}$. $/ \mathrm{km}^{2}$.

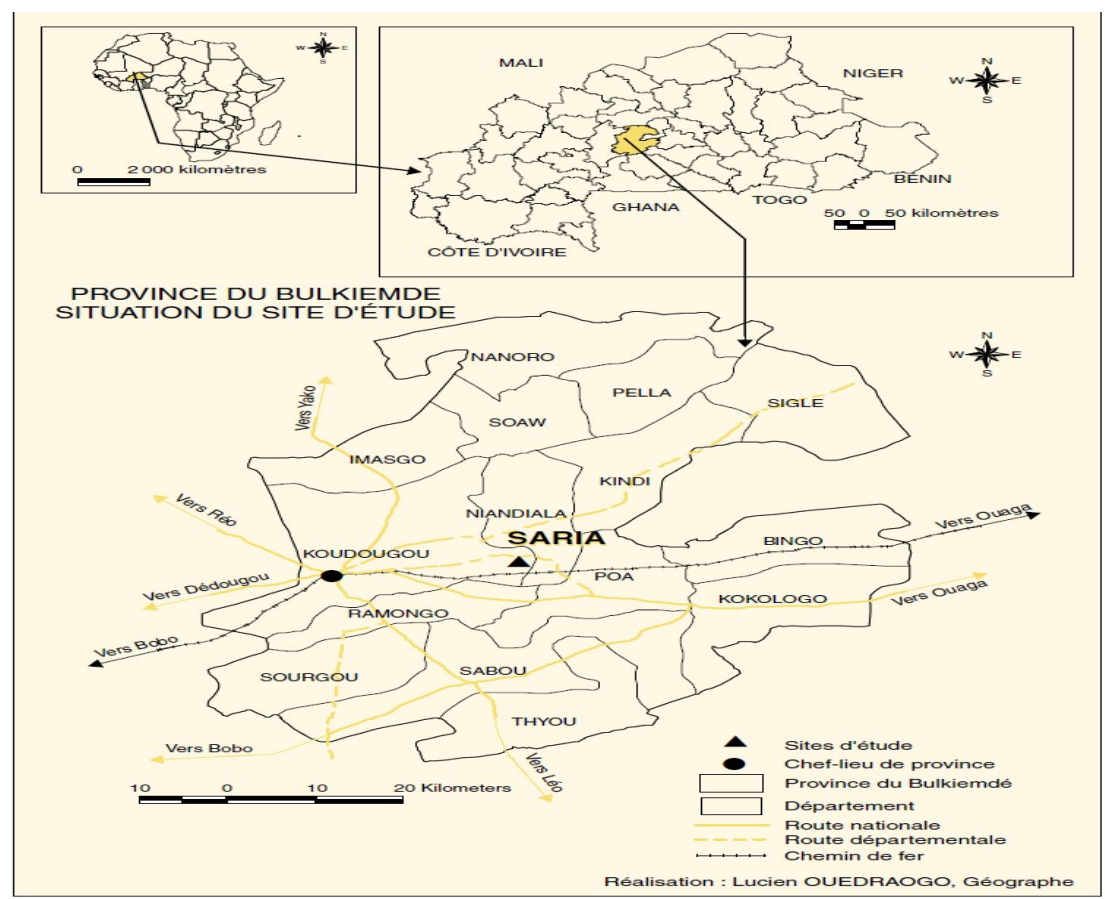

Figure 1: Situation de la zone d'étude Méthodologie

Détermination de la biomasse aérienne de $P$. reticulatum: La détermination de la biomasse 
aérienne a été réalisée par la méthode directe. Elle a consisté à abattre et peser les arbres afin d'obtenir leur biomasse réelle. Cette méthode, bien que destructrice et difficile à réaliser à grande échelle, reste de loin la meilleure méthode d'estimation de la biomasse des arbres (Walker et Désaère, 2004 ; CIRAD et FAO 2012; Yussufu, 2013). Au cours de cette étude, la biomasse épigée de $P$. reticulatum en fonction de l'âge et des réservoirs de l'arbuste a été déterminée. A cet effet, quatre-vingt (80) pieds dont dix (10) de quinze (15) ans, trente-cinq (35) de cinq (05) ans et trente-cinq (35) de trois (03) ans ont été abattus. Ces pieds ont été choisis de manière aléatoire parmi les pieds des plantations équiennes. Avant l'abattage, les paramètres dendrométriques suivants ont été mesurés : le diamètre à $0,20 \mathrm{~cm}$ du sol à l'aide d'un ruban mou, la hauteur totale à l'aide d'une perche, le diamètre du houppier dans deux directions à l'aide d'un décamètre. Pour les pieds à plusieurs tiges, chaque tige a été mesurée ensuite la circonférence unique de l'arbre a été obtenue en faisant la racine carrée de la somme de chaque circonférence élevée au carré (Koala et al., 2017) :

$\mathrm{C}_{0,1}=\sqrt{\sum_{i=1}^{n} c_{i}^{2}}$ Sachant que :

- $\quad n=$ nombre de tiges;

- $\quad C_{i}=$ circonférence à $0,1 \mathrm{~m}$ pour la tige $\mathrm{i}(\mathrm{cm})$
- $\quad$ C $0,1=$ circonférence à $0,1 \mathrm{~m}$ de l'individu (cm).

Après abattage, le tronc, les branches, les feuilles et les fruits (s'il y en a) ont été séparées. La biomasse fraiche de chaque réservoir (tronc, branches, brindilles, feuilles et fruits) a été déterminée par pesée sur place à l'aide d'une balance (Sartorius ISO 9001). Cette méthode a été utilisée avec succès par de nombreux auteurs pour déterminer la biomasse des différents réservoirs de l'arbre (Walker et Desanker, 2004 ; Jacques et Saugier, 2008 ; Yussufu, 2013). Après la pesée sur place à l'état frais des arbres, les échantillons (aliquotes) de chacun des réservoirs, également pesés sur place ont été prélevés. Pour chaque arbre, cinq (05) échantillons de $500 \mathrm{~g}$ de chaque réservoir ont été prélevés aléatoirement. Ces échantillons ont été séchés à l'étuve (CIRAD et FAO 2012; Yussufu, 2013). Les parties ligneuses ont été séchées à $105^{\circ} \mathrm{C}$ (pendant environ une semaine, jusqu'à masse constante) et les parties non ligneuses séchées à $70^{\circ} \mathrm{C}$ (pendant environ trois jours, jusqu'à masse constante). Une fois secs, ces échantillons ont été pesés à nouveau, à l'aide d'une balance (Mettler PM 4000g, $d=0,01$, e $=0,1$ ) pour obtenir la masse sèche pour chacun des compartiments de chaque arbre et par la suite pour chaque arbre selon la méthode de Jacques et Saugier (2008). Quelques étapes de la méthodologie sont illustrées par la figure 2. 


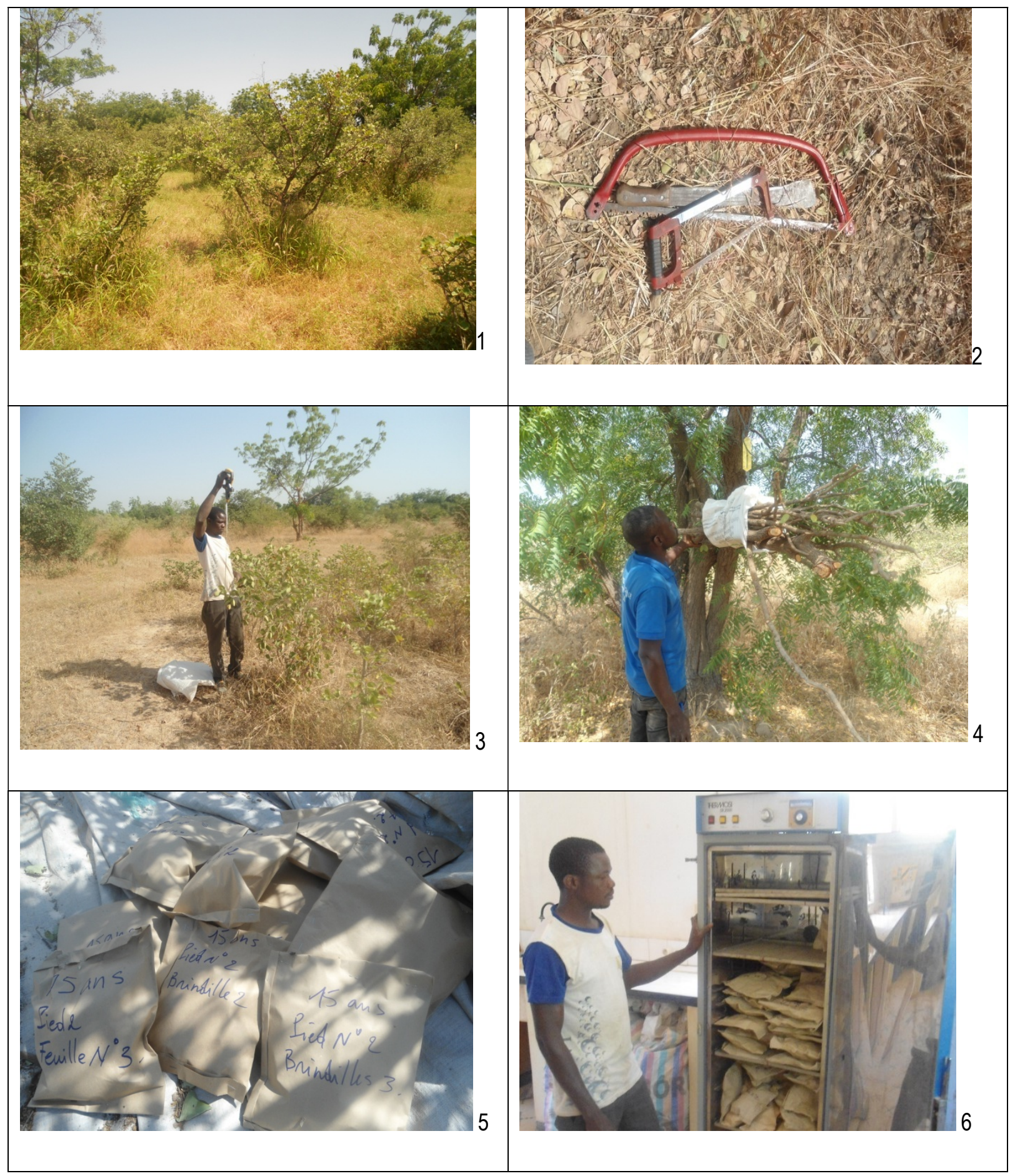

Figure 2 : lllustrations de quelques étapes de la méthode a- Plantation de $P$. reticulatum ; b- Quelques équipements de terrain ; c-Mesure de la hauteur totale d'un pied de 5 ans, d- pesée des branches ; e- échantillons conditionnés pour être envoyés au laboratoire ; f- Séchage des échantillons à l'étuve) 


\section{Analyse des données}

Biomasses sèches: Les biomasses sèches des différents compartiments de l'arbuste et la biomasse sèche totale du Pied ont été obtenues par les différentes formules suivantes (CIRAD et FAO, 2012):

$$
\begin{aligned}
& \text { - le taux d'humidité de l'échantillon } \\
& \mathrm{Xi}=\frac{\text { Baliquote sèche } i}{\text { Baliquote fraiche } i} \\
& \text { - le taux d'humidité moyen du compartiment } \\
& \mathbf{X m}=\frac{\sum X i}{n} \\
& \text { - la biomasse sèche du compartiment } \\
& \text { Bs }=\boldsymbol{X} \boldsymbol{m} \times \boldsymbol{B} \boldsymbol{f} \\
& \text { - la biomasse sèche total du pied } \\
& \text { Bst }=\text { Bs tronc }+ \text { Bs Branches }+ \text { Bs Brindilles }+ \text { Bs } \\
& \text { Feuilles } \\
& \text { - la biomasse sèche moyenne d'un pied de } P \text {. }
\end{aligned}
$$

Avec :

- $\quad$ Xi le taux d'humidité de l'échantillon ; l'aliquote $\mathrm{i}$;

- $\quad$ B aliquote fraiche $\mathrm{i}$ la biomasse fraiche de l'aliquote i ;

- $\quad X m$ le taux d'humidité moyen du compartiment ;

- $\quad$ Bf la biomasse fraîche du compartiment ;

- Bms la biomasse moyenne d'un pied de $P$. reticulatum à un âge donné et

- $\quad n$ le nombre d'échantillon $(n=5)$ dans le cas de cette étude.

- $\quad \quad \quad \quad$ nombre de pieds

La biomasse moyenne d'un peuplement de $P$. reticulatum à l'hectare a été obtenue en multipliant la biomasse d'un pied par le nombre théorique de pieds à l'hectare (ce nombre est 1100 pieds pour une plantation à $3 \times 3 \mathrm{~m}$ ). Cette biomasse a été déterminée pour chaque âge concerné ( 3,5 et 15 ans) par cette étude

Quantités de carbone: De nombreux auteurs estiment que la quantité de carbone d'un arbre correspond en moyenne à la moitié de sa masse sèche (Walker et Desanker, 2004 ; Jacques et Saugier, 2008). Ainsi, les masses sèches recueillies ont toutes été rapportées en quantité de carbone. Pour ce faire, les masses sèches mesurées ou estimées ont été multipliées par 0,5, conformément au ratio par défaut de teneur en $C$ de la masse sèche ligneuse recommandée (Lufala et al., 2008). Les quantités de carbone ont donc été calculées de la manière suivante (Bakayoko et al., 2007 ; Defrenet, 2012) :

$\mathrm{Cb}=\mathrm{Bs} \times \mathrm{Tc}$

- $\quad$ Cb la quantité de stock de carbone du réservoir,

- Bs la biomasse sèche du réservoir et

- Tc le taux de carbone du réservoir Le stock de carbone par pied s'est fait en sommant les stocks de carbone des différents réservoirs du pied:

$\mathrm{Cbt}=\mathrm{Cb}$ tronc $+\mathrm{Cb}$ branches $+\mathrm{Cb}$ brindilles $+\mathrm{Cb}$ feuilles $+\mathrm{Cb}$ fruits (pour les pieds qui en portent) Le stock de carbone moyen d'un pied de $P$. reticulatum à un âge a été déterminé par la formule suivante:

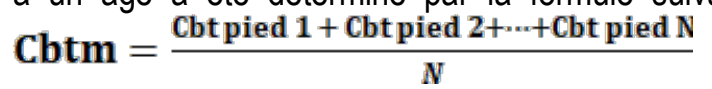

Le stock de carbone à l'hectare a été estimé en multipliant le stock de carbone moyen d'un pied par le nombre de pieds à l'hectare.

tC ha $^{-1}=$ Cbtm $\times 1100$

- $\quad$ tC ha-1 la quantité de carbone à l'hectare,

- $\quad$ Cbtm la quantité de carbone moyen stockée par un pied et

- $\quad 1100$ le nombre théorique de pieds de $P$. reticulatum pour une plantation à des écartements de 3 $\times 3 \mathrm{~m}$

La capacité de stockage de carbone de la biomasse aérienne en tonnes de carbone par hectare et par an a été déterminée par la formule : $\mathrm{tC} \mathrm{ha}^{-1} \mathrm{an}^{-1}=\mathrm{tC}^{\mathrm{t}} \mathrm{ha}^{-1} \mathrm{x}$ $A^{-1}$

- tC ha-1 an-1 la capacité de stockage de carbone de la biomasse aérienne en tonnes de carbone par hectare et par an ;

- $\quad$ C ha-1 la quantité de carbone à l'hectare ;

- $\quad$ A est l'âge de la plantation.

Etablissement des relations allométriques : Selon le principe de base de l'allométrie, les proportions entre hauteur et diamètre, entre taille du houppier et diamètre, entre biomasse et diamètre, obéissent à une règle qui est la même pour tous les arbres vivants dans les mêmes conditions, du plus petit au plus gros (Adamou et Chimène, 2008 ; CIRAD et FAO, 2012). C'est ce principe qui permet de prédire une mensuration d'un arbre (typiquement sa biomasse) en fonction d'une autre mensuration (par exemple son diamètre). Généralement, les relations allométriques sont établies à partir du diamètre à hauteur de poitrine ou de la hauteur des arbres (M'bow, 2009). Selon CIRAD et FAO (2012), la production d'un peuplement monospécifique et équienne d'une essence donnée, dans une région donnée, est complètement déterminée 
par sa hauteur moyenne. Cette étude correspond au cas ci-dessus évoqué. Ainsi l'établissement des relations allométriques au cours de cette étude est parti des hauteurs moyennes des peuplements de $P$. reticulatum. Ce choix se justifie aussi par le fait que dans les peuplements de cinq (05) et trois (03) ans, les pieds de $P$. reticulatum n'ont pas encore atteint la hauteur de $1,30 \mathrm{~m}$. En plus des régressions développées à partir de la hauteur totale du pied, des régressions ont été développées à partir de la surface du houppier et de la circonférence à la base du tronc.

Des modèles de tarifs ont été ajustés. L'ajustement d'un modèle consiste à estimer les paramètres de ce modèle à partir de données. Les modèles qui ont été ajustés sont les suivants (CIRAD et FAO, 2012) :

$$
\begin{array}{ll}
\text { - } & \text { Exponentiel : } Y=a e^{b x} \\
\text { - } & \text { Logarithmique : } Y=a \ln (x)-b \\
\text { - } & \text { Polynomial : } Y=a x^{2}+b x+c \\
\text { - } & \text { Puissance : } Y=a x^{b}
\end{array}
$$

Avec:

- Yla biomasse,

- $\quad a$ et $b$ des constantes à déterminer et

- $\quad x$ est le paramètre dendrométrique utilisé pour ajuster le modèle (hauteur totale, surface du houppier, circonférence du tronc à $0,10 \mathrm{~m}$ du sol).

Un concept clé de l'ajustement des modèles est la détermination des paramètres de précision et d'optimisation comme le coefficient de détermination $\left(R^{2}\right)$ et les résidus du modèle. Le coefficient de

\section{RESULTATS}

Paramètres dendrométriques: Les paramètres dendrométriques des pieds de $P$. reticulatum qui ont fait l'objet de cette étude sont présentés dans le tableau 1. La hauteur moyenne d'un pied de 15 ans est de 3,06 m $\pm 0,43 \mathrm{~m}$, elle est de $1,62 \mathrm{~m} \pm 0,40 \mathrm{~m}$ pour les individus de 5 ans et de $0,45 \mathrm{~m} \pm 0,14 \mathrm{~m}$ pour ceux de 3 ans. Quant à la circonférence moyenne du tronc à 10 $\mathrm{cm}$ du sol, elle varie de $34,90 \mathrm{~cm} \pm 11,98 \mathrm{~cm}$ (individus de 15 ans) à $3,4 \pm 0,70 \mathrm{~cm}$ (individus de 3 ans). Le diamètre moyen du houppier est de $3,98 \pm 0,79 \mathrm{~m}$ pour les individus de 15 ans, il est de 1,08 $\pm 0,36 \mathrm{~m}$ et de $0,38 \pm 0,06 \mathrm{~m}$ respectivement pour les pieds de 5 ans et de 3 ans. Le plus grand accroissement annuel en hauteur est obtenu pour les individus de 5 ans $(0,32 \pm$ détermination $\left(\mathrm{R}^{2}\right)$ mesure la qualité de l'ajustement. II s'interprète comme le ratio de la variance expliquée par le modèle sur la variance totale. II est compris entre 0 et 1 et plus il est proche de 1, plus la qualité de l'ajustement est meilleure.

Le résidu, ou erreur résiduelle, est l'écart entre la valeur observée de la variable réponse et sa prédiction. Le résidu pour chaque observation est calculé par la formule suivante :

$\varepsilon_{\mathrm{i}}=\mathrm{Bi}-\dot{\mathrm{B}}_{\mathrm{i}}$ :

- $\quad$ Avec $\varepsilon_{i}$ le résidu pour le pied $\mathrm{i}$,

- B B la biomasse réelle du pied i et

- $\quad \dot{B}_{i}$ la biomasse estimée à partir de l'équation d'allométrie pour le pied $i$

L'ajustement d'un modèle est d'autant meilleur que ses résidus sont faibles.

Analyses statistiques : Une analyse de variance à un facteur (ANOVA) suivie du test des moindres carrés de Fischer a été réalisée, afin de comparer les moyennes des différents paramètres dendrométriques, des quantités de matières sèches et de carbone stockées par $P$. reticulatum au seuil de $5 \%$. Aussi, les biomasses obtenues par mesure directe et celles issues des différents modèles ont été soumises à une ANOVA pour évaluer la performance de chaque estimateur de biomasse. Les analyses statistiques ont été réalisées à l'aide du logiciel statistique Xlstat (version 14.3.07, Addinsoft, 2007)

0,07 m) (Tableau 2). C'est également au niveau de ces individus de 5 ans que la plus grande croissance en circonférence du tronc à $10 \mathrm{~cm}$ du sol est obtenue soit $2,96 \mathrm{~cm} \pm 0,53 \mathrm{~cm}$. Cet accroissement est de 2,32 $\mathrm{cm} \pm$ $0,80 \mathrm{~cm}$ pour les pieds de 15 ans et de $1,13 \mathrm{~cm} \pm 0,23$ $\mathrm{cm}$ pour ceux de 3 ans. Quant à l'accroissement annuel du diamètre du houppier, il est plus important pour les pieds de 15 ans avec une valeur de 0,26 cm $\pm 0,05 \mathrm{~m}$. Cette valeur n'est pas significativement différente de celle obtenue pour les pieds de 5 ans $(0,21 \pm 0,07 \mathrm{~m})$. Par contre, l'accroissement moyen annuel en diamètre pour les pieds de 3 ans est significativement plus bas $(0,12 \pm 0,02 \mathrm{~m})$. 
Tableau 1: Paramètres dendrométriques moyens des pieds de $P$. reticulatum

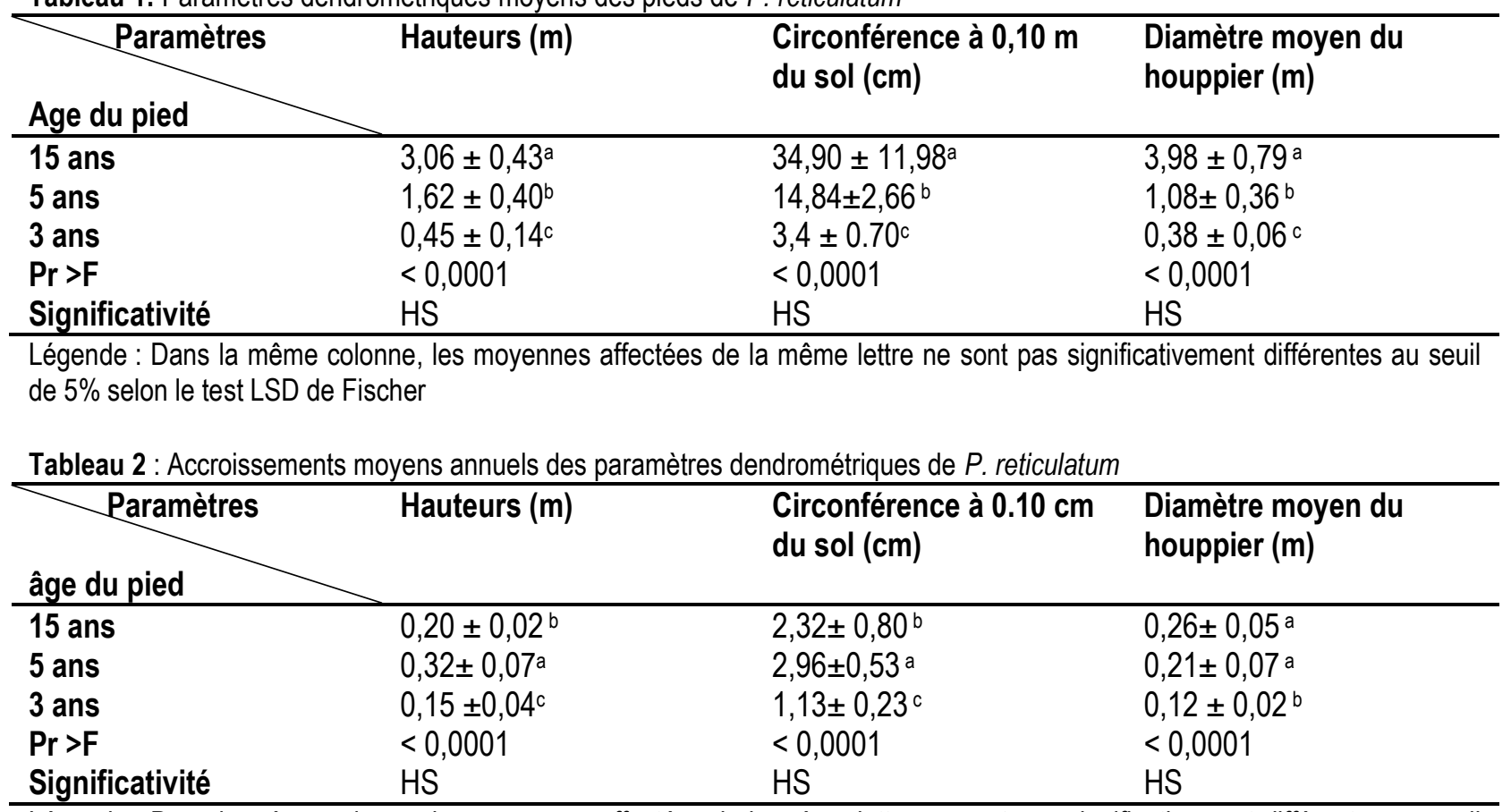

Légende : Dans la même colonne, les moyennes affectées de la même lettre ne sont pas significativement différentes au seuil de $5 \%$ selon le test LSD de Fischer

Biomasses aériennes et quantités de carbone stockées

Répartition de la biomasse aérienne suivant les compartiments: Au niveau des pieds de 15 ans, ce sont les branches qui ont la plus grande biomasse sèche $(55 \%)$, suivies des brindilles (18\%) (Figure 3). Les feuilles et le tronc représentent respectivement $14 \%$ et $13 \%$ de la biomasse sèche. Chez les individus de 5 ans, c'est le tronc qui renferme la plus grande quantité de matière sèche (31\%). Les branches et les brindilles représentent respectivement $29 \%$ et $20 \%$ de la biomasse sèche. Pour ce qui est de la répartition de la biomasse sèche entre les différents compartiments des pieds de 3 ans, ce sont les feuilles qui ont la plus grande proportion (45\%). Le tronc renferme $24 \%$ de la biomasse sèche d'un pied de $P$. reticulatum de 3 ans. Les branches et les brindilles ont des proportions respectives de $17 \%$ et $14 \%$.

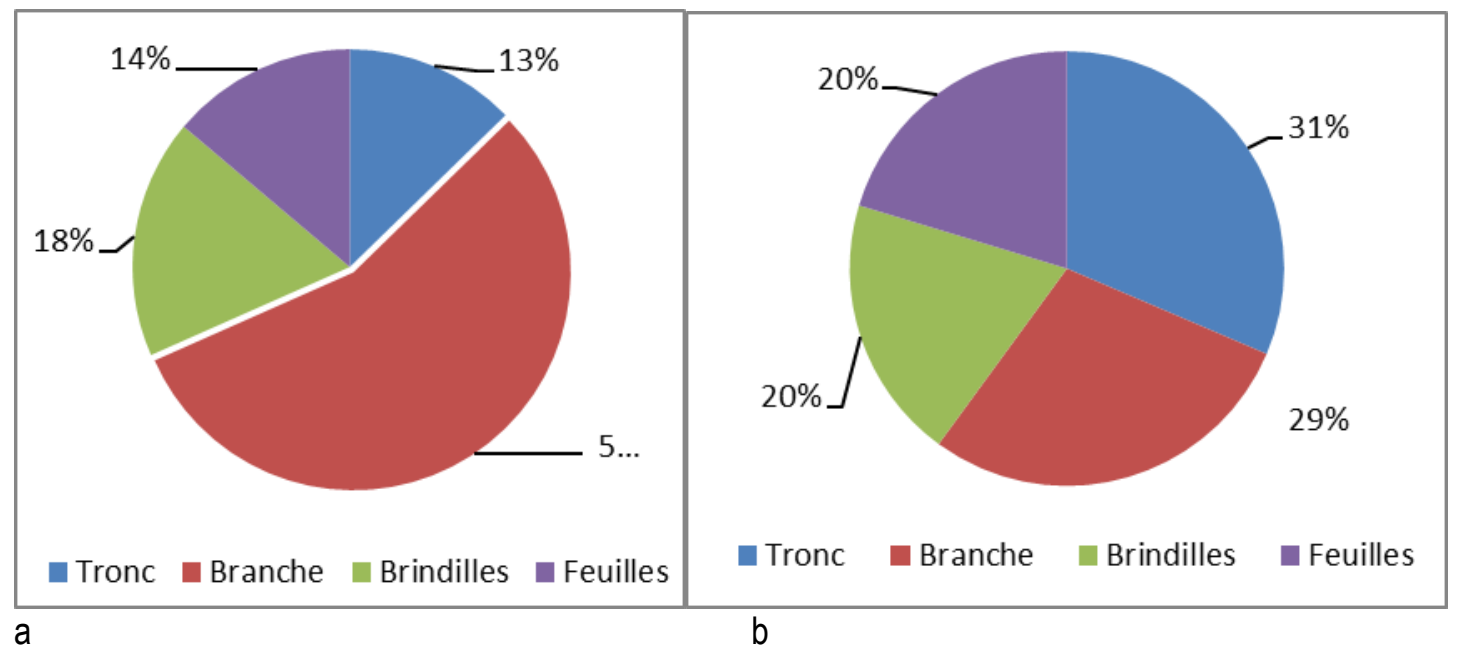




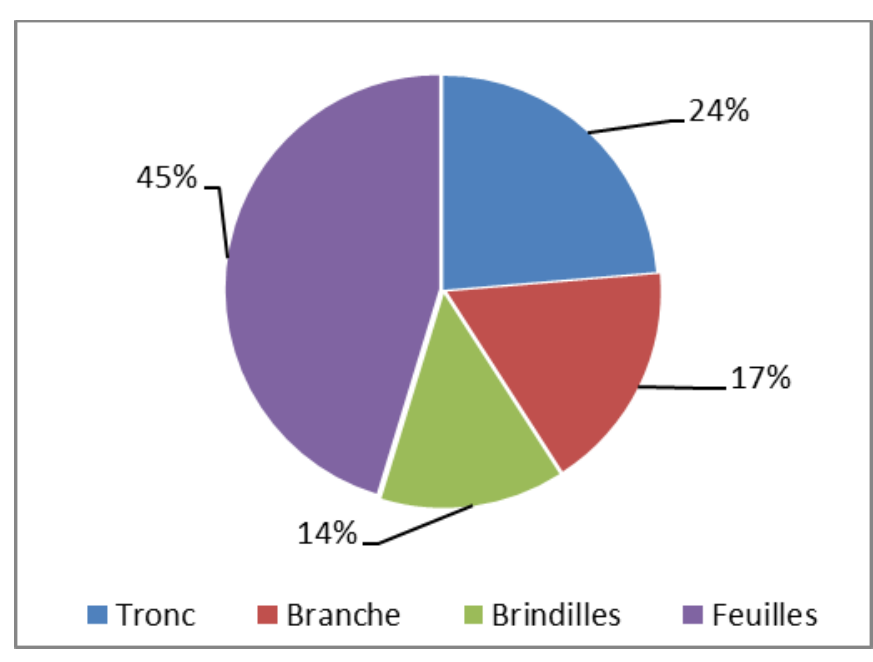

C

Figure 3 : Répartition de la biomasse entre les différents compartiments du pied de $P$. reticulatum $a$ : pied de 15 ans, $b$ : pieds de 5 ans, $c$; pieds de 3 ans

Quantités de Biomasses et de carbone stockées par $P$. reticulatum : Au bout de 15 ans une plantation de $P$. reticulatum, d'écartement $3 \mathrm{~m} \times 3 \mathrm{~m}$ stocke en moyenne à l'hectare $30 \pm 6,49$ tonnes de matières sèches. Cette matière sèche renferme environ $15 \pm$ 3,24 tonnes de carbone (Tableau 3). Cette quantité est significativement supérieure à celle stockée au bout de 5 ans $(1,82 \pm 0,67)$. La quantité de carbone stockée à l'hectare au bout de 3 ans est de 0,014 $\pm 0,007$ tonne. La capacité de stockage de carbone de la biomasse aérienne en tonnes de carbone par hectare et par an varie en fonction de l'âge de la plantation. Pour une plantation de 15 ans elle est de $1,00 \pm 0,21 \mathrm{tC} \mathrm{ha}^{-1} \mathrm{an}^{-1}$. Elle est de 0,36 $\pm 0,13$ et 0,005 $\pm 0,002 \mathrm{tC} \mathrm{ha-1}^{-1} \mathrm{an}^{-1}$ respectivement pour une plantation de 5 ans et celle de 3 ans.

Tableau 3 : Biomasses et quantités de carbone

\begin{tabular}{|c|c|c|c|c|}
\hline Age du pied & $\begin{array}{l}\text { Biomasses } \\
\text { aériennes à ha } \\
\left(\mathrm{tMS} \mathrm{ha}{ }^{-1}\right)\end{array}$ & 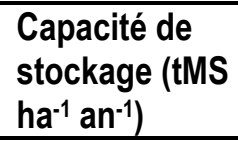 & $\begin{array}{l}\text { Carbone totaux } \\
\text { stockés }\left(\mathrm{tC} \mathrm{ha}^{-1}\right)\end{array}$ & $\begin{array}{l}\text { Capacité de stockage } \\
\left.\text { de carbone (tC ha-1 } \mathrm{an}^{-1}\right)\end{array}$ \\
\hline 15 ans & $30 \pm 6,49 a$ & $2 \pm 0,43^{a}$ & $15,00 \pm 3,24^{a}$ & $1,00 \pm 0,21^{a}$ \\
\hline 5 ans & $3,65 \pm 1,34^{b}$ & $0,72 \pm 0,27 \mathrm{~b}$ & $1,82 \pm 0,67 \mathrm{~b}$ & $0,36 \pm 0,13^{b}$ \\
\hline 3 ans & $0,03 \pm 0,01^{c}$ & $0,01 \pm 0,004 c$ & $0,014 \pm 0,007 c$ & $0,005 \pm 0,002^{c}$ \\
\hline $\mathrm{Pr}>\mathrm{F}$ & $<0,0001$ & $<0,0001$ & $<0,0001$ & $<0,0001$ \\
\hline Significativité & HS & HS & HS & $\mathrm{H}$ \\
\hline
\end{tabular}

Légende : Dans la même colonne, les moyennes affectées de la même lettre ne sont pas significativement différentes au seuil de $5 \%$ selon le test LSD de Fischer

tMS ha-1 : tonne de matière sèche à l'hectare, tC ha -1 : tonne de carbone à l'hectare

Dans la même colonne, les moyennes affectées de la même lettre ne sont pas significativement différentes au seuil de $5 \%$ selon le test LSD de Fischer

\section{Relations allométriques}

Relations allométriques développées pour les pieds

de 15 ans: Les régressions développées pour les pieds de 15 ans montrent des performances très peu variées (Tableau 4). En effet, on observe des coefficients de détermination $\left(R^{2}\right)$ variant peu et tous supérieurs à 0,9. Les représentions de quelques régressions sont présentées par la figure 4. L'analyse graphique de ces courbes indique que le modèle polynomial explique mieux les variations de la biomasse sèche des pieds de 15 ans de $P$. reticulatum en fonction des mesures dendrométriques. 
Tableau 4 : Modèles calculés à partir de la hauteur et leur $R^{2}(n=80$ individus)

\begin{tabular}{lll}
\hline Modèles & Formules & $\mathbf{R}^{2}$ \\
\hline \multirow{3}{*}{ Exponentiel } & 15 ans $: Y=11,36 \mathrm{e}^{0,327 x}$ & 0,945 \\
& 5 ans $: Y=1,359 \mathrm{e}^{0,608 x}$ & 0,798 \\
& 3 ans $: Y=0,009 \mathrm{e}^{2,179 x}$ & 0,942 \\
\hline \multirow{3}{*}{ Logarithmique } & 15 ans $: Y=32,04 \ln (x)-4,321$ & 0,967 \\
& 5 ans $: Y=0,298 \ln (X)+2,171$ & 0,831 \\
\hline \multirow{3}{*}{ Polynomial } & 3 ans $Y=0,028 \ln (x)+0,050$ & 0,919 \\
\hline \multirow{3}{*}{ Puissance } & 15 ans $: Y=-1,422 x^{2}+19,28 x-14,22$ & 0,968 \\
& 5 ans $: Y=-0,458 x^{2}+3,590 x-0,867$ & 0,830 \\
& 3 ans $Y=0,019 x^{2}+0,042 x+0,003$ & 0,949 \\
\hline
\end{tabular}

Légende $: \mathrm{Y}=$ Biomasse sèche en $\mathrm{Kg}, \mathrm{X}=$ hauteur du pied en $\mathrm{m}$

Relations allométriques développées pour les pieds de 05 ans : II existe une certaine corrélation entre la biomasse sèche totale des pieds de $P$. reticulatum âgés de 05 ans et leurs mesures dendrométriques. Les coefficients de déterminations varient entre 0,79 et 0,87 (Tableau 5). La corrélation entre la biomasse sèche et les mesures dendrométriques n'est pas très forte. Cependant la part de la variabilité de la biomasse sèche qui est expliquée par la variabilité des paramètres dendrométriques est supérieure ou égale à $80 \%$ pour tous les modèles développés. Les courbes des ajustements renseignent que c'est le modèle polynomial qui explique mieux la variabilité de la biomasse en fonction des mesures de l'arbre (Figure 5).

Tableau 5 : Modèles calculés à partir de la surface du houppier et leur $R^{2}(n=80$ individus $)$

\begin{tabular}{lll}
\hline Modèles & Formules & $\mathbf{R}^{\mathbf{2}}$ \\
\hline \multirow{3}{*}{ Exponentiel } & 15 ans $: Y=10,95 \mathrm{e}^{0,062 x}$ & 0,920 \\
& 5 ans : $Y=1,458 \mathrm{e}^{0,705 \mathrm{x}}$ & 0,825 \\
& 3 ans $: Y=0,011 \mathrm{e}^{7,262 x}$ & 0,751 \\
\hline \multirow{3}{*}{ Logarithmique } & 15 ans $: Y=33,68 \ln (x)-63,38$ & 0,954 \\
& 5 ans $: Y=2,922 \ln (X)+2,997$ & 0,841 \\
& 3 ans $Y=0,024 \ln (x)+0,082$ & 0,770 \\
\hline \multirow{3}{*}{ Polynomial } & 15 ans $: Y=-0,099 x^{2}+5,392 x-30,81$ & 0,957 \\
& 5 ans $: Y=-0,514 x^{2}+3,724 x-0,231$ & 0,844 \\
\hline \multirow{2}{*}{ Puissance } & 3 ans $Y=0,249 x^{2}+0,146 x+0,008$ & 0,789 \\
& 15 ans $: Y=1,512 x^{1,074}$ & 0,939 \\
& 5 ans $: Y=2,966 x^{0,852}$ & 0,843 \\
\hline
\end{tabular}

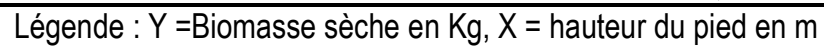




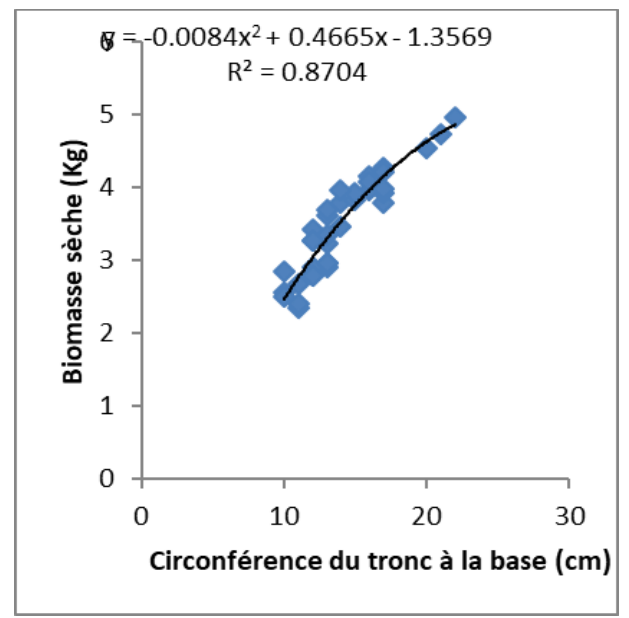

a

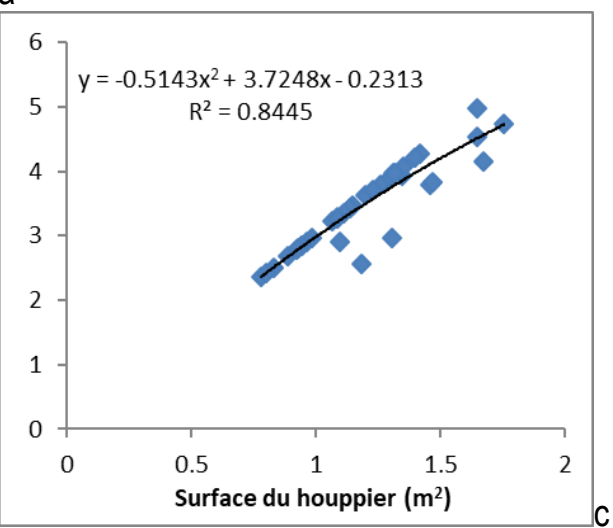

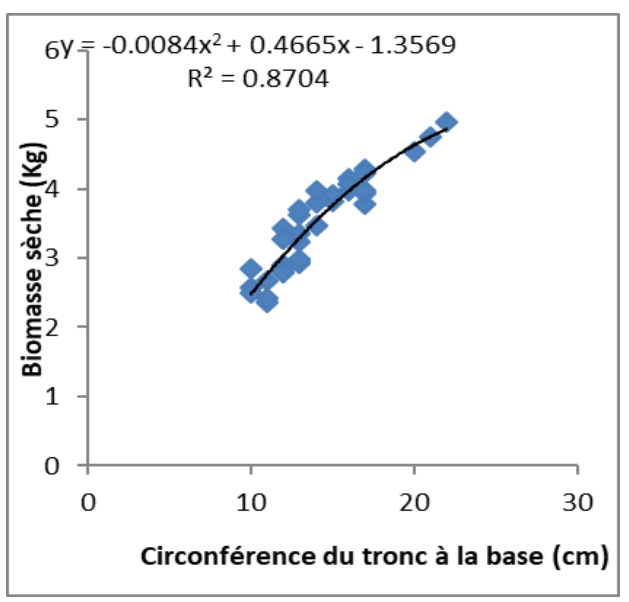

b

Figure 5: Evolution de la biomasse sèche en fonction des paramètres dendrométriques pour les pieds de 05 ans $a$ : modèle logarithmique à partir de la hauteur des pieds ; $b$ : modèle polynomial à partir de la circonférence à la base du tronc ; $c$ : modèle polynomial à partir de la surface du houppier.

Relations allométriques développées pour les pieds de $\mathbf{0 5}$ ans : La corrélation est également forte entre les paramètres dendrométriques et la biomasse épigée des pieds de 03 ans de $P$. reticulatum (Tableau 6). Ici aussi, c'est le modèle polynomial qui explique mieux les variabilités de la biomasse en fonction des paramètres dendrométriques (Figure 6).

Tableau 6 : Modèles calculés à partir de la circonférence du tronc à la base et leur $R^{2}(n=80$ individus)

\begin{tabular}{lll}
\hline Modèles & Formules & $\mathbf{R}^{\mathbf{2}}$ \\
\hline \multirow{3}{*}{ Exponentiel } & 15 ans $: Y=11,43 \mathrm{e}^{0,025 x}$ & 0,920 \\
& 5 ans $: Y=1,483 \mathrm{e}^{0,059 x}$ & 0,804 \\
& 3 ans $: Y=0,009 \mathrm{e}^{0,199 x}$ & 0,899 \\
\hline \multirow{3}{*}{ Logarithmique } & 15 ans $: Y=30,62 \ln (x)-80,65$ & 0,916 \\
& 5 ans $: Y=3,123 \ln (X)-4,716$ & 0,869 \\
\hline \multirow{3}{*}{ Polynomial } & 3 ans $Y=0,028 \ln (x)-0,018$ & 0,877 \\
& 15 ans $: Y=0,249 x^{2}+0,146 x+0,008$ & 0,922 \\
& 5 ans $: Y=0,008 x^{2}+0,466 x-1,356$ & 0,870 \\
Puissance & 3 ans $Y=0,000 x^{2}+0,004 x+0,002$ & 0,916 \\
& 15 ans $: Y=0,833 x^{0,969}$ & 0,916 \\
& 5 ans $: Y=0,328 x^{0,892}$ & 0,837 \\
\hline
\end{tabular}

Légende $: Y=$ Biomasse sèche en $\mathrm{Kg}, \mathrm{X}=$ hauteur du pied en $\mathrm{m}$ 


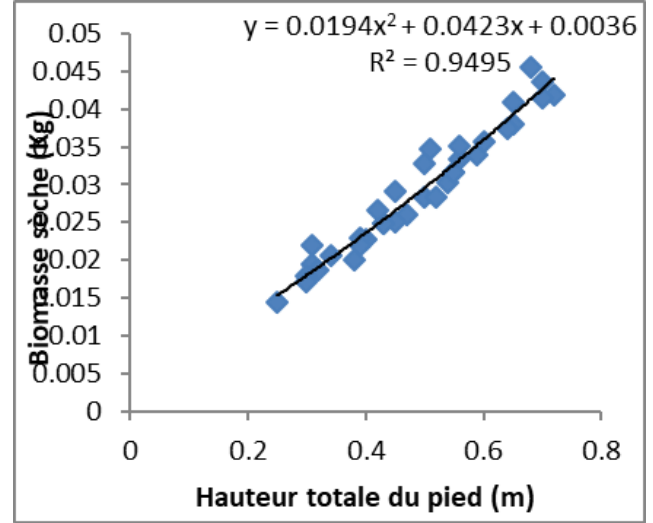

a

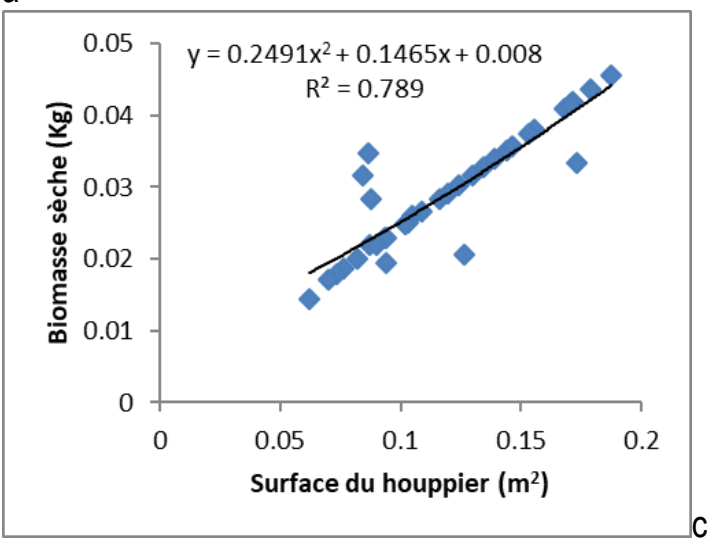

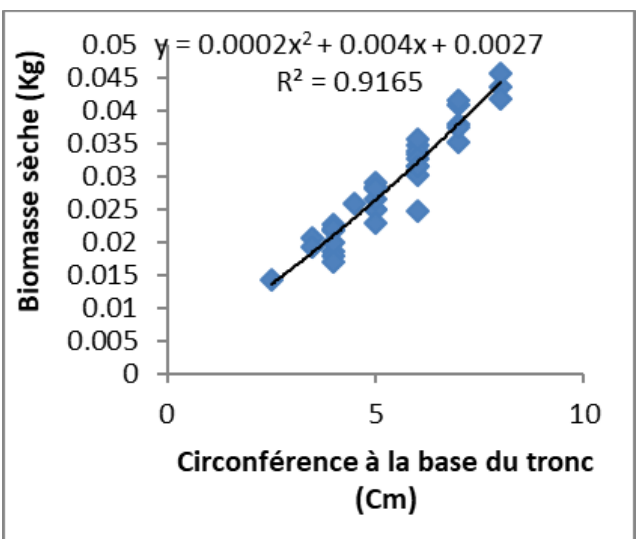

b

Figure 6: Evolution de la biomasse sèche en fonction des paramètres dendrométriques pour les pieds de 03 ans $a$ : : modèle polynomial à partir de la hauteur des pieds, $b$ : modèle polynomial à partir de la circonférence à la base du tronc, $c$ : modèle polynomial à partir de la surface du houppier

Evaluation des modèles développés : Le coefficient de détermination est une première forme d'évaluation de la performance d'un modèle. La comparaison des biomasses mesurées et estimées en est une autre. L'analyse des biomasses estimées par les modèles et les biomasses mesurées sur le terrain indique que les estimateurs de biomasse établis sont très performants.
En effet, pour la majorité des modèles, il n'existe aucune différence significative entre les biomasses mesurées et celles évaluées à l'aide des équations allométriques (Tableau 7,81 et 9 ). Seul le modèle polynomial développé à partir de la hauteur des pieds de 03 ans indique des différences significatives avec les biomasses mesurées sur le terrain.

Tableau 7 : Comparaison des biomasses estimées et mesurées (tMS/ha- ${ }^{-1}$ ) pour les pieds de 15 ans

\begin{tabular}{|c|c|c|}
\hline Modèles & Biomasse sèche & Résidus \\
\hline biomasse mesurée & $30,003 \pm 6,49^{a}$ & \\
\hline$Y=-1,422(\mathrm{H} \mathrm{cm})^{2+19,28(H)-14,22}$ & $34,299 \pm 4,9$ a & $-4,29 \pm 5,55^{a}$ \\
\hline$Y=11,43 e^{0,02^{*} \mathrm{C} 0,10}$ & $33,51 \pm 0,11 \mathrm{~b}$ & $-4,29 \pm 4,61^{a}$ \\
\hline$Y=10,95 e^{0,062}$ & $34,58 \pm 0,39^{b}$ & $-4,58 \pm 4,63^{a}$ \\
\hline
\end{tabular}

\begin{tabular}{lll}
\hline $\operatorname{Pr}>\mathbf{F}$ & $>0,22$ & 0,941 \\
\hline Significativité & NS & NS \\
\hline $\begin{array}{l}\text { Légende : Dans la même colonne, les moyennes affectées de la même lettre ne sont pas significativement différentes au seuil } \\
\text { de 5\% selon le test LSD de Fischer }\end{array}$
\end{tabular}


Tableau 8 : Comparaison des biomasses estimées et mesurées (tMS/ha- ${ }^{-1}$ ) pour les pieds de 05 ans

\begin{tabular}{|c|c|c|}
\hline Modèles & Biomasse sèche & Résidus \\
\hline biomasse mesurée & $3,822 \pm 0,75^{a}$ & \\
\hline$Y=-3,29 \ln (H)+2,171$ & $3,822 \pm 0,69$ a & $0,023 \pm 0,36^{a}$ \\
\hline$Y=-0,008 C^{2}+0,466 C-1,356$ & $3,90 \pm 0,73 a$ & $0,000 \pm 0,29 a$ \\
\hline$Y=-0,514 \mathrm{SH}^{2}+3,724 \mathrm{SH}-0,2313$ & $3,822 \pm 0,69 a$ & $-0,075 \pm 0,34^{a}$ \\
\hline
\end{tabular}

\begin{tabular}{lll}
\hline $\operatorname{Pr}>\mathbf{F}$ & $>0,711$ & 0,435 \\
\hline Significativité & NS & NS \\
\hline $\begin{array}{l}\text { Légende : Dans la même colonne, les moyennes affectées de la même lettre ne sont pas significativement différentes au seuil } \\
\text { de } 5 \% \text { selon le test LSD de Fischer }\end{array}$
\end{tabular}

Tableau 9: Comparaison des biomasses estimées et mesurées ( $t M S / h^{-1}$ ) pour les pieds de 03ans

\begin{tabular}{|c|c|c|}
\hline Modèles & Biomasse sèche & Résidus \\
\hline biomasse mesurée & $0,032 \pm 0,009^{b}$ & \\
\hline$Y=-0,06 H^{1,013}$ & $0,032 \pm 0,008^{b}$ & $0,000 \pm 0,003^{a}$ \\
\hline$Y=0,249 S^{2}+0,146 \mathrm{SH}+0,008$ & $0,069 \pm 0,03^{a}$ & $0,006 \pm 0,002^{a}$ \\
\hline$Y=0,004 C+0,002$ & $0,026 \pm 0,006^{b}$ & $-0,037 \pm 0,002^{b}$ \\
\hline
\end{tabular}

\begin{tabular}{ll}
\hline $\operatorname{Pr}>\mathrm{F}$ & $>0,24$ \\
\hline Significativité & S \\
\hline $\begin{array}{l}\text { Légende : Dans la même colonne, les moyennes affectées de la même lettre ne sont pas significativement différentes au seuil } \\
\text { de } 5 \% \text { selon le test LSD de Fischer }\end{array}$
\end{tabular}

\section{DISCUSSION}

Paramètres dendrométriques: Les plus grandes mesures dendrométriques sont obtenues avec les pieds les plus âgés. En effet, les pieds de 15 ans mesurent environ $3 \mathrm{~m}$ de hauteur pour une circonférence à la base du tronc de $34,90 \mathrm{~cm}$ et un diamètre du houppier de $3,98 \mathrm{~m}$. Cependant, ces pieds n'ont pas atteint leur taille maximale. Arbonnier (2009) indique que $P$. reticulatum peut atteindre $10 \mathrm{~m}$ de hauteur. Louppe et Ouattara (1996) ont trouvé à Korhogo (Côte d'Ivoire) une hauteur moyenne de 1,49 $\mathrm{m}$ pour une plantation de $P$. reticulatum âgée de 4,5 ans. Ces résultats et ceux de la présente étude sont semblables (1,62 $\mathrm{m}$ pour des pieds de 5 ans). Joet et al., (1998) trouvent au Niger sur sol sablo limono argileux hydromorphes $4 \mathrm{~m}$ de hauteur pour des pieds de 5 ans et $8 \mathrm{~m}$ pour des pieds de 8 ans. La pluviosité annuelle de leur site d'étude varie entre 300 et $400 \mathrm{~mm}$. Les résultats de leur étude et ceux de la présente étude ne sont pas en accord. Cela pourrait s'expliquer par le fait que les pieds de $P$. reticulatum qui ont fait l'objet de leur étude sont issus de régénération naturelle assistée tandis que ceux de la présente étude sont issus de plantation. Les pieds issus de régénération naturelle croîtraient donc plus vite que ceux issus de plantation. Les pieds provenant de rejets de souche dispose d'un système racinaire déjà développé qui facilite la nutrition minérale tandis que pour les pieds provenant de plantation, il faut du temps pour que le système racinaire s'installe. Une analyse des taux d'accroissements des pieds de $P$. reticulatum de différents âges permet de confirmer ceci. En effet, le taux d'accroissement annuel en hauteur des pieds de 3 ans est inférieur à celui des pieds de 5 ans. Une autre explication de la meilleure croissance rapportée par Joet et al., (1998) serait une adéquation entre le type de sol de leur milieu d'étude et les exigences édaphiques de l'espèce. L'écologie de $P$. reticulatum indique qu'il préfère les sols lourds et mal drainés (Arbonnier, 2009).

Biomasses et quantités de carbone : La quantité de carbone exprimée en tC/ha est proportionnelle à la phytomasse, car cette quantité a été calculée en 
multipliant la masse de la végétation par la teneur en carbone. II s'en suit que la quantité de carbone et la masse de la végétation évoluent dans le même sens. Les valeurs trouvées au cours de la présente étude sont $15 \mathrm{tC} /$ ha pour la plantation de 15 ans, 1,86 et 0,014 tC/ha respectivement pour les plantations de 5 et de 3 ans. Adamou et Chimène (2008) ont trouvé au Cameroun $0,66 \mathrm{tC} /$ ha stockée par diverses espèces d'arbustes dans une savane arbustive. Ces quantités sont inférieures à celles obtenues au cours de la présente étude pour les pieds de 15 ans et de 5 ans mais supérieures à celles des pieds de 3 ans de la présente étude. Cela s'explique par le fait que les sujets ayant fait objet de leur étude sont des arbustes dont le diamètre et la hauteur varient respectivement de 1,5 à $14,6 \mathrm{~cm}$ et de 0,45 à $1,95 \mathrm{~m}$. Ces pieds sont donc plus petits que les pieds de $P$. reticulatum de 15 ans et plus grands que les pieds de 05 ans et de 3 ans de la présente étude. Ainsi, la quantité de carbone stockée par une formation végétale est fonction des paramètres dendrométriques des arbres qui la constituent. Cette quantité est grande pour les grands arbres et petite pour les petits arbres. Bayen et al., (2015) en zone soudanienne du Burkina Faso ont estimé à $0,33 \mathrm{tC} / \mathrm{ha}$ la quantité de carbone séquestré par une plantation de Jatropha curcas âgée de 4 ans. Cette quantité est nettement inférieure à celle obtenue pour les pieds de $P$. reticulatum de 5 ans $(1,82 \mathrm{tc} / \mathrm{ha})$ mais supérieure à celle obtenue pour les pieds de $P$. reticulatum de 3 ans $(0,014 \mathrm{tc} / \mathrm{ha})$. Les faibles quantités de carbone stockées par les pieds de $P$. reticulatum de 3 ans s'expliquent par le fait que ce sont de très petits pieds qui ne sont pas encore affranchis. En effet, ils consacrent plus d'énergie à la mise en place d'un système racinaire fort pour assurer leur croissance ultérieure comme l'indiquent Diédhiou et al. (2016). M'bow (2009) estime entre 3 et $7 \mathrm{tC} /$ ha la quantité de carbone stockée par la forêt Classée de Wélor (Sénégal). Cette quantité est inférieure à celle stockée par nos plantations de $P$. reticulatum de 15 ans $(15,00$ $\pm 3,24 \mathrm{tC} / \mathrm{ha}$ ). Les quantités de carbone stockées par $P$. reticulatum sont supérieures à celles de divers arbustes des savanes soudano-sahéliennes. En effet Lufala et al., (2009) lors de leur étude sur les potentiels de stockage de carbone de certains arbustes ont trouvé en moyenne un stock de $0,66 \mathrm{~kg} \mathrm{C} /$ arbuste de Guiera senegalensis. Cette valeur est inférieure aux valeurs trouvées pour les pieds de $P$. reticulatum de 15 ans $(12,63 \pm 4,53)$ et ceux de 5 ans $(1,11 \pm 0,13)$. Thiam (1998) a trouvé un stock de 0,86 tC ha-1 pour Acacia Senegal, cette valeur est inférieure à celle obtenue pour les plantations de $P$. reticulatum de 15 ans $(15,00$ $\left.\pm 3,24 \mathrm{tC} \mathrm{ha}^{-1}\right)$ et de 5 ans $\left(1,82 \pm 0,67 \mathrm{tC}^{\mathrm{ha}} \mathrm{h}^{-1}\right)$. Ainsi, $P$. reticulatum possède un fort potentiel de séquestration de carbone. Cela s'explique par le fait que $P$. reticulatum est un arbuste massif qui croit rapidement et produit une abondante biomasse foliaire (Yélemou et al., 2013 ; Arbonnier, 2009).

Relations allométriques : La majorité des régressions développées ont un coefficient de détermination avoisinant 90\%. Rappelons que le coefficient de détermination indique dans le cas de cette étude la part de la variabilité de la biomasse sèche qui est expliqué par la variabilité des paramètres dendrométriques. L'analyse des coefficients de détermination et la comparaison des biomasses mesurées et des biomasses estimées indiquent que la circonférence à la base du tronc ne semble pas être un bon paramètre à utiliser pour des régressions de la biomasse de $P$. reticulatum. C'est ce qui justifierait que Lufala et al., (2008), en développant des régressions pour estimer la biomasse d'une espèce arbustive (Guiera senegalensis) ont utilisé le diamètre moyen du houppier. D'après Peltier et al. (2007), il y a un fort lien entre les propriétés de la canopée et la biomasse épigée. Les résultats de la présente étude sont en accord avec ceux de Peltier et al. (2007), en effet les meilleurs modèles sont développés à partir de la surface du houppier. Le coefficient de détermination à lui seul ne permet pas la validation d'un modèle. II faut en plus effectuer des analyses des résidus des modèles. Selon CIRAD et FAO (2012), les meilleurs modèles sont ceux dont les résidus sont faibles présentant ainsi de très faibles différences entre les biomasses estimées à partir des modèles testés et les biomasses estimées à partir des mesures de terrain. II n'existe pas de différences significatives entre les biomasses estimées à partir des modèles testés et les biomasses estimées à partir des mesures de terrain pour les pieds de 15 ans, il en est de même pour les pieds de 05 ans. Cependant pour les pieds de 03 ans, parmi les modèles testés, le modèle polynomial développé à partir de la hauteur surestime la biomasse. II n'existe donc pas de corrélation de type polynomiale entre la biomasse épigée sèche et la hauteur total des pieds de 03 ans. Cela pourrait s'expliquer par le fait que les pieds de 03 ans sont de petits sujets qui ne sont pas encore affranchis. En effet, les jeunes pieds de $P$. reticulatum cherchent d'abord à installer leurs racines avant de croitre en hauteur (Yélemou, 2010).Selon la prescription de M'bow (2009), aucune équation ne doit être utilisée pour estimer la biomasse si le paramètre 
dendrométrique des sujets dépasse l'intervalle indiqué. La remarque de Saint André (2005) et Kenzo (2009) selon laquelle les équations allométriques pour prédire des biomasses sont généralement très satisfaisantes

\section{CONCLUSION}

Des relations allométriques ont été développées à partir de la hauteur totale, de la surface du houppier et de la circonférence du tronc. Au total, 15 équations ont été développées. L'évaluation de ces modèles a permis de retenir trois. Ces résultats pourront servir à ajuster les calculs de biomasse et de carbone stockés par $P$. reticulatum au lieu d'emprunter des modèles conçus pour d'autres espèces et d'autres types de formations

\section{REFFERENCES}

Adamou I. et Chimène A. F., 2008. Estimation du stock de carbone dans les faciès arborés et arbustifs des savanes soudano-guinéennes de Ngaoundéré, Cameroun. Cameroon Journal of Experimental Biology. 04 (01): 1-11.

Addinsoft (2007). Xlsat. https://www.xlstat.com.

Arbonnier M., 2009. Arbres, arbustes et lianes des zones sèches d'Afrique de l'Ouest. MNHNQUAE, France, $576 \mathrm{p}$.

Bakayoko O., Mathieu A. A., Brahima C., et Dossahoua T., 2007. Carbon storage in biomass and soils of four Australian Acacia stands of Anguededou station (South-East of Cote d'Ivoire). European Journal of Scientific Research. 19 (1): 25-37.

Bayen P., Bognounou F., Lykke M. A., Ouédraogo M. et Thiombiano A., 2015. The use of biomass production and allometric models to estimate carbon sequestration of Jatropha Curcas L. plantations in western Burkina Faso. Environ Dev Sustain. 17 : 30-42.

Boulmane M., Makhloufi M., Bouillet J. P., Saint-André L., Satrani B., Halim M., El Antry S., 2010. Estimation du stock de carbone organique dans les Quercus ilex du Moyen Atlas Marocain. Acta bot. Gallica. 157 : 451-467.

CIRAD et FAO, 2012. Manuel de construction d'équations allométriques pour l'estimation du volume et de la biomasse des arbres: de la mesure de terrain à la prédiction. Organisation des Nations Unies pour l'Alimentation et l'Agriculture, Rome (Italie), $220 \mathrm{p}$.

Defrenet E., 2012. Evaluation de la biomasse et de la productivité souterraines du caféier dans un système agroforestier au Costa Rica. Mémoire pour les futaies forestières et permettent d'estimer des biomasses à partir de mesures simples et rapides est vérifier pour cette étude

végétales. Les résultats indiquent qu'il existe un fort lien entre la quantité de biomasse épigée de $P$. reticulatum et certaines mesures dendrométriques telles que la surface du houppier. En outre l'espèce a une forte capacité de séquestration de carbone comparativement à d'autres espèces arbustives. Au regard de ces résultats, il faut encourager la plantation et/ou la régénération naturelle assistée de l'espèce.

de Master en productions végétales. Agrocampus Ouest, France, $37 \mathrm{p}$.

Diédhou I., Diallo D., Mbengue A., Hernandez R. R., Bayala R., Diéme R., Diédhiou P. M., Sène A., 2016. Allometric equations and carbon stocks in tree biomass of Jatropha curcas $L$. in Senegal's Peanut Basin. Biomass and Bioenergy, $21 \mathrm{p}$.

Hansein J., 2004. Defusing the global warming time bomb. Sci. Am. 290 : 68-77.

IPCC 2007. Bilan 2007. Des Changements climatiques, rapport du groupe d'experts intergouvernemental sur l'évolution du climat, rapport de synthèse. $[E n$ ligne : http://www.ipcc.ch/pdf/assessmentreport/ar4/syr/ar4_syr_fr.pdf (Accès le 04/07/2017)]

Jacques, G. et Saugier, B. 2008. Les puits de carbone, Editions Tec \& Doc, Lavoisier, Paris, $171 \mathrm{p}$.

Joet A., Jouve P., Banouin M., 1998. Le défrichement amélioré au sahel : une pratique adoptée par les paysans. Bois et forêts des tropiques. 255 (1): 3-43.

Kenzo, T. 2009. Development of allometric relationships for accurate estimation of above and below-ground biomass in tropical secondary forests in Sarawak, Malaysia. Journal of Tropical Ecology: 371-386.

Koala J., Sawadogo L., Savadogo P., Aynekulu E., Heiskanen J., Saïd M., 2017. Allometric equations for below-ground biomass of four key woody species in West African savannawoodlands. Silva Fennica. 51 (3) : 1-15.

Lufala A., Diedhiou N. A. S., Séné M., Kizito F., Dick R. P. et Noller J. S., 2009. Allometric 
relationships and peak-season community biomass stocks of native shrubs in Senegal's peanut basin. Journal of Arid Environments, $73(3):$ : 260-266.

M'bow C., 2009. Potentiel et dynamique des stocks de carbone des savanes soudaniennes et soudano-guinéennes du Sénégal. Thèse de Doctorat d'Etat, Université Cheikh Anta Diop de Dakar, Dakar, $311 \mathrm{p}$.

Ministère de l'Economie et des Finances, 2009. Recensement Général de la Population et de l'Habitation de 2006 (RGPH-2006), Résultats définitifs, rapport de synthèse des rapports d'analyses. $66 \mathrm{p}$.

Ouédraogo A., 2006. Diversité et dynamique de la végétation ligneuse de la partie orientale $d u$ Burkina Faso. Thèse de doctorat unique, Université de Ouagadougou, Burkina Faso, $195 p$

Peltier R., Forkong C. N., Ntoupka M., Manlay R., Henry M., Morillon V., 2007. Evaluation du stock de carbone et de la productivité en bois d'un parc à Karité du Nord-Cameroun. Bois et forêts des tropiques. 39-50.

Saint André L., 2005. Age related equations for above and below- ground biomass of a Eucalyptus hybrid in Congo. Forest Ecology and Management 205:199-214

Sanou S., 2005. Piliostigma reticulatum (D.C.) Hochst : Potentialités fourragères et essai d'amélioration de la valeur nutritive des gousses. Mémoire d'Ingénieur du Développement Rural, Institut du Développement Rural, Université Polytechnique de Bobo Dioulasso, Burkina Faso, $57 \mathrm{p}$.

Thiam S., 1998. Contraintes liées aux pratiques de gestion des ressources naturelles au Sénégal. Mémoire de master, Université Abdou MOUMOUNI de Niamey, Niger, $65 \mathrm{p}$.

Tyano A., 2016. Impact des formations à Piliostigma reticulatum (DC.) Hochst sur la régénération de la végétation et les propriétés chimiques et biologiques du sol en zone soudanienne du Burkina Faso. Mémoire d'ingénieur des Sciences de l'Environnement et du Développement Rural option Eaux, Forêts et
Environnement, Centre Universitaire Polytechnique de Dédougou, Université de Ouagadougou, Burkina Faso, 66 p.

Tyano A., Yélémou B. et Bationo B. A., 2016. Impacts of Piliostigma reticulatum on chemical and biological properties of fallows in sudanian zone of Burkina Faso. International Journal of Current Research 8 (11): 41878-41885.

Walker, S. M. et Desanker P.V., 2004. The impact of land use on soil carbon in Miombo Woodlands of Malawi. Forest Ecology and Management 203: 345-360.

Yélemou B. 2010. Biologie et écologie des espèces du genre Piliostigma et leur contribution à la dynamique de la matière organique des sols en zone sahélo-soudanienne au Burkina Faso. Mémoire Thèse unique de Doctorat, Université de Ouagadougou, Burkina Faso, $150 p$

Yélemou B., Yaméogo G., Millogo-Rasolodimby J., et Hien V., 2007. Germination sexuée et dynamique de développement de Piliostigma reticulatum (D.C.) Hochst, une espèce agroforestière du Burkina Faso, Sécheresse 18 (3) : 185-192.

Yélemou B., Yaméogo G., Bationo B. A., MillogoRasolodimby J. et Hien V., 2012. Les formations à Piliostigma en zone sahélosoudanienne du Burkina Faso : Etat des peuplements, dynamique de la régénération sexuée et pression anthropique. Int. J. Biol. Chem. Sci. 6 (5) : 2083-2096.

Yélemou B., Sawadogo P., Traoré S., MillogoRasolodimby J. et HIEN V., 2015. Floristic diversity of Piliostigma associations in relation to latitudinal gradient, soil and climate variables in Burkina Faso, West Africa. Tropical Ecology 56 (1): 57-76.

Yélémou B., Tyano A., Bationo B.A., Ouattara B., Koala J. et Rasolodimby M. J., 2017. Effects of $P$. reticulatum on the vegetation dynamics in Sudanian zone of Burkina Faso. Journal of Plant Studies. 6 (2): 77- 93.

Yussufu E. K., 2013. Carbone stocké dans un essai de provenances d'Acacia mangium à Ibi Village sur le plateau des Batéké en République Démocratique du Congo. Mémoire de Maitre ès Sciences, Université Laval, 89 p. 


\section{Liste des photographies}

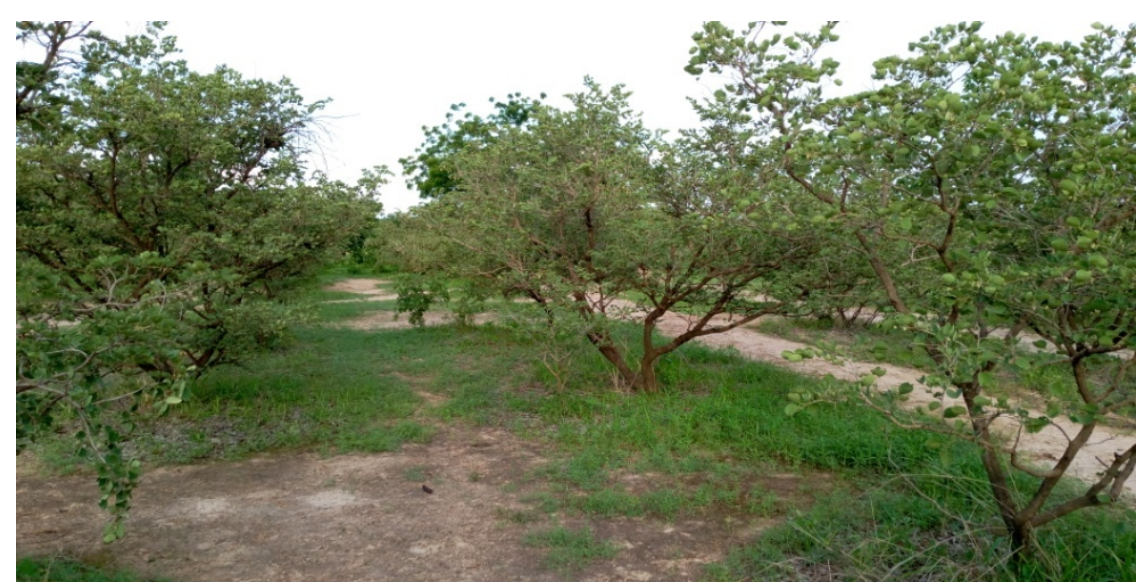

Photo 1 : Plantation de $P$. reticulatum de 15 ans 